\title{
Levantamento epidemiológico da prática de atividade física na cidade de São Paulo
}

\author{
Marco Túlio de Mello ${ }^{1}$, Ana Cláudia Fernandez² e Sérgio Tufik ${ }^{3}$
}

\section{RESUMO}

Estudos epidemiológicos conduzidos em geral ou em populações específicas são de vital importância para a determinação e implementação de políticas públicas. Um dos problemas de saúde mais comuns que causa grande preocupação é o crescente número de pessoas obesas e, conseqüentemente, os problemas de saúde advindos da obesidade. No entanto, as desordens de sono, como a insônia, e distúrbios no ciclo vigília-sono que resultam de fatores psicológicos, neurológicos e sociais são também importantes problemas de saúde. Neste estudo, foi realizado um levantamento epidemiológico da prática de atividade física e problemas de sono na população geral da cidade de São Paulo. Mil indivíduos de todas a regiões da cidade, representando todas as classes sociais, responderam a um questionário específico. Os resultados mostraram que somente $31,3 \%$ dos entrevistados estão engajados em algum tipo de atividade física e somente $36,4 \%$ desses indivíduos têm supervisão de um profissional qualificado. Os distúrbios de sono associados com a insônia foram reportados por $27,1 \%$ dos indivíduos fisicamente ativos e $35,9 \%$ dos não ativos ( $\mathrm{p}<0,003)$. Dados de um estudo nacional em saúde e nutrição mostram um aumento na percentagem de indivíduos obesos no Brasil. Isto pode ser parcialmente explicado pelos nossos achados de baixos níveis de atividade física desta população pesquisada, o que pode levar a um aumento na incidência de doenças cardíacas e metabólicas (diabetes). Os resultados revelaram uma baixa percentagem de indivíduos engajados em atividade física regular

1. Universidade Federal de Uberlândia/UFU-FAEFI/NIFEP.

2. Departmento de Pediatria da Universidade Federal de São Paulo (UNIFESP-EPM).

3. Departmento de Psicobiologia da Universidade Federal de São Paulo (UNIFESP-EPM).

Endereço para correspondência:

Marco Túlio de Mello

Departamento de Psicobiologia

Universidade Federal de São Paulo - EPM

Rua Napoleão de Barros, 925 - Vila Clementino

04020-002 - São Paulo, SP - Brasil

Fax: (+55-11) 572-5092

E-mail: tmello@psicobio.epm.bre tmello@ufu.br

Rev Bras Med Esporte - Vol. 6, № 4 - J ul/ A go, 2000 sob supervisão da população em geral. Portanto, é importante alertar a população quanto aos benefícios da prática regular e supervisionada de exercícios físicos.

Palavras-chave: Atividade física. Desordens de sono. Condicionamento físico. Sono. Estudos epidemiológicos.

\section{ABSTRACT}

\section{Epidemiological survey of the practice of physical activi- ty in São Paulo City}

Epidemiological studies conducted on general or specific populations are of vital importance for the determination of public health policies. One of the current health problems causing most concern is the growing number of obese people and, consequently, the health problems that result from obesity. In addition, sleep disorders, such as insomnia and disruption of the wake-sleep cycle that result from psychological, neurological and social factors are also important health problems. In the present study, we carried out an epidemiological survey of the practice of physical activity and of sleep problems in the general population of São Paulo City, Brazil. One thousand subjects from all regions of the City and representing all social categories answered a specific questionnaire. The findings showed that only $31.3 \%$ of the interviewees engaged in some type of physical activities and only $36.4 \%$ of these did so under professional supervision. Sleep complaints associated with insomnia were reported by $27.1 \%$ and $35.9 \%$ of physically active and inactive subjects $(p<0.03)$, respectively. Data from the National Survey on Health and Nutrition show an increase in the percentage of obese individuals in Brazil. This could partly be explained by our findings of low levels of physical activity, which could ultimately lead to an augmented incidence of metabolic (diabetes) and cardiac diseases. The present findings revealed a low percentage of engagement in supervised physical activities by the general population. Therefore, it becomes important to alert the population to the benefits of practicing supervised physical exercises.

Key words: Physical activity. Sleep disorders. Fitness. Sleep. Epidemiological surveys. 


\section{INTRODUÇÃO}

A inatividade pode ser descrita como um estado em que o movimento corporal é mínimo. Em termos de gasto energético a inatividade representa um gasto energético que está muito próximo da taxa metabólica basal ${ }^{1}$. Muitos estudos indicam que a diminuição do gasto energético precede ou acompanha o desenvolvimento da obesidade, tanto em crianças como em adultos ${ }^{2-5}$.

A falta de atividade física pode ter efeitos negativos sobre a vida do indivíduo como: aumento da taxa de diabetes, aparecimento de doenças cardíacas, e leva até mesmo a um aumento do risco de enfarte ${ }^{6}$. De acordo com estatísticas norte-americanas, um estilo de vida sedentária é o contribuinte nas mortes por doenças crônicas, incluindo doenças coronárias, enfarte e câncer colorretal, perdendo somente para o hábito de fumar e obesidade ${ }^{7}$. A prevalência de uma estilo de vida sedentário em adultos nos Estados Unidos tem aumentado gradualmente nas duas últimas décadas, em ambos os sexos, a percentagem de indivíduos que não praticam exercícios regulares aumenta com a idade e diminui com o nível de renda familiar 6 .

$\mathrm{O}$ interesse em estudos a respeito do papel da atividade física na prevenção de doenças cardíacas tem aumentado na América do Norte, Austrália e Nova Zelândia ${ }^{8}$. Dentre as formas de atividade física realizadas no tempo livre, andar é a mais comum, metade das pessoas que realizam atividade física em seu tempo livre andam para se exercitarem, e a maioria dessas pessoas andam regularmente, de acordo com estudo realizado por Siegel et al. ${ }^{9} \mathrm{em} 45$ Estados norte-americanos em 1990 com um total de 81.557 participantes que responderam a questionário específico.

$\mathrm{O}$ uso de questionários para quantificar a atividade física tem sido a maneira mais prática e mais usada em estudos epidemiológicos sobre a taxa de realização de atividade física de uma dada população, mesmo tendo-se em consideração que este método pode apresentar deficiências referentes a tipos de perguntas e manipulação involuntária das respostas ${ }^{8}$.

Segundo estudo realizado nos Estados Unidos e Canadá, entre 1972 e 1983, os indivíduos mais jovens e aqueles de condição socioeconômica mais alta são mais ativos em seu tempo livre e os homens apresentam maior envolvimento com esportes e exercícios vigorosos. Os dados demonstram que $20 \%$ dos participantes realizavam atividades físicas para alcançar benefícios cardiovasculares e $40 \%$ se exercitavam de modo moderado, enquanto que $40 \%$ eram sedentários. A diferença entre os sexos foi maior em relação a atividades físicas vigorosas, mas desaparece quando se trata de atividade física moderada ${ }^{10}$. Em estudo realizado no Canadá ${ }^{11}, 58 \%$ dos adultos acima de 20 anos de ida- de foram considerados sedentários e $17 \%$ eram pouco ativos. Vinte e cinco por cento se exercitavam regularmente e, desses, os homens eram 7\% mais ativos que as mulheres. Este estudo ainda mostrou que a atividade física é mais freqüente entre os mais jovens, seguida de um progressivo declínio e um subsequiente aumento aos 50 anos de idade.

De acordo com estudo realizado com 6.814 homens e mulheres, a inatividade atinge $32 \%$ da população da Austrália, $54 \%$ são moderadamente ativos e $15 \%$ são descritos como realizando atividades aeróbias ${ }^{12}$. Em um estudo mais recente foi demonstrado que dos 17.053 participantes, $29 \%$ eram sedentários, e estes eram constituídos na sua maioria por indivíduos mais velhos, com menos instrução e tendo menor renda ${ }^{13}$.

A relação entre atividade física e sono foi examinada em uma população urbana somente em indivíduos adultos. A análise dos dados ressaltou de um modo geral que a prática da atividade física é um fator favorável à melhora da qualidade do sono, tendo como itens analisados a latência de sono, profundidade do sono e o alerta matutino. Tal estudo sugere que o exercício deva ser realizado em horários que não estejam próximos aos períodos de dormir, no intuito de não provocar outras desordens como insônia e diminuição da eficiência do sono ${ }^{14}$. Também tem sido sugerido que fatores psicossociais, assim como o estilo de vida, local em que se dorme e as condições as quais os indivíduos vivem, influenciam diretamente na qualidade do sono e na performance física ${ }^{15}$.

Mulheres sedentárias submetidas a um treinamento físico de 12 semanas e uma média diária de 60 minutos a $70 \%$ do $\dot{\mathrm{VO}}_{2}$ máx. apresentaram um aumento do estágio 2 do sono NREM e uma diminuição dos estágios 3 e 4 (sono delta/sono de ondas lentas - SWS). Os autores comentaram que esses resultados podem ter sido influenciados pelo estresse físico e psicológico produzidos pela atividade física intensa. Entretanto, os dados da literatura não suportam essa hipótese ${ }^{16}$ em uma revisão bibliográfica, Trinder et $a l .{ }^{17}$ observaram que há alterações no padrão de sono após a prática de atividade física. Um dos pontos importantes observados neste estudo foi o aumento do tempo total de sono e tempo de sono nos estágios 3 e 4 . A discussão do trabalho gira em torno da diferença existente entre a resposta ao exercício físico agudo que proporciona alterações nos estágios de sono delta e a atividade física crônica que não traz mudança no padrão de sono, causado pelo processo de adaptação do organismo humano. Recentemente, Youngstedt et al. ${ }^{18}$ elaboraram uma síntese quantitativa sobre o efeito do exercício agudo no sono, utilizando uma revisão bibliográfica que visou analisar a influência da atividade física sobre alguns parâmetros do sono. Foi estudada a latência de sono, estágio 2 , sono de ondas lentas, sono 
REM, latência de sono REM e insônia de início de noite. A quantificação dos estudos relatou uma diminuição do sono REM e um aumento na latência desse mesmo estágio do sono. Foi encontrado um aumento no sono de ondas lentas, mas em uma quantidade pequena de exercícios não promoveu alteração significante. $\mathrm{O}$ aumento do tempo total de sono foi proporcional ao volume das atividades desenvolvidas e as variáveis como sono de ondas lentas, REM, latência de REM e tempo total de sono sofreram efeitos classificados pelos autores de pequenos a moderados. Uma limitação apresentada nesta análise é de tais estudos serem realizados em pessoas que no geral são designados de "bons dormidores", isto de acordo com os autores gera uma incógnita sobre os resultados entre pessoas que têm problemas de sono e indivíduos normais.

Apesar da importância da atividade física regular na qualidade de vida, não existem muitos estudos epidemiológi$\cos$, a respeito da atividade física na população brasileira. Então, o objetivo deste estudo foi o de realizar um levantamento epidemiológico da atividade física da população da cidade de São Paulo e cruzar os dados obtidos com a qualidade e o padrão de sono dessa população estudada.

\section{MÉTODOS}

Segundo Cavalheiro ${ }^{19}$, existem duas fontes onde podem ser obtidas informações no campo da saúde: os estabelecimentos onde as pessoas vão buscar assistência médica e a própria comunidade. Um levantamento de condições de saúde por entrevistas domiciliares é um método largamente empregado, tanto para a investigação epidemiológica exclusiva quanto para levantamento contínuo das considerações da saúde da comunidade.

O presente estudo utilizou para a coleta dos dados um questionário elaborado e validado por Del Giglio ${ }^{20}$ que tem por objetivo avaliar o padrão e as queixas de sono, acrescido de quatro questões simples sobre prática e tipo de atividade física realizada pelo indivíduo. Este questionário consiste de 96 questões sobre distúrbios do sono, ciclo vigíliasono e contém ainda 4 questões sobre a prática de atividade física.

O questionário foi aplicado na cidade de São Paulo e a amostra foi dividida em 3 fases, de acordo com critério adotado por especialistas em desenho amostral. O critério para a determinação da amostra foi probabilístico: realizou-se, inicialmente, o sorteio dos subdistritos e setores censitários e, dentre eles, um sorteio de domicílios através de um intervalo fixo para cada setor. Finalmente, um indivíduo foi sorteado em cada casa ou apartamento. Para o sorteio dos subdistritos foi considerada a divisão da Cidade de São Paulo em regiões homogêneas de acordo com a alocação da população economicamente ativa ${ }^{21}$. Nessa divisão, apenas os subdistritos da Capital foram considerados. Assim, os diferentes setores de cada subdistrito (fornecidos pela Fundação Instituto Brasileiro de Geografia e Estatística - IBGE) e listados em ordem decrescente, em relação ao nível médio de renda e sorteados com um intervalo fixo, de tal forma, que as diferentes faixas estivessem representadas. Esse intervalo foi obtido mediante o quociente do número total de domicílios no setor (casas mais apartamentos) pelo número proposto de 25 entrevistas por setor. Antes de ser efetuado os sorteio dos setores, foram excluídos dos domicílios coletivos como: hospitais, quartéis, asilos, orfanatos, hotéis, alojamentos de trabalhadores e escolas.

A amostra foi constituída de 1.000 entrevistas probabilísticas. As entrevistas foram realizadas em residências com pessoas acima de 20 anos até a lucidez, de ambos os sexos, nas classes sociais A, B, C, D e E (classificação social descrita pela ABIPEME - Associação Brasileira de Institutos de Pesquisa de Mercado) ${ }^{22}$.

Os critérios de aplicação das entrevistas no campo foram os seguintes:

a) Determinar a priori que seriam sorteados 25 domicílios no máximo por setor, independente da extensão geográfica do mesmo; b) Considerar "informantes qualificados" pessoas da família com mais de 15 anos. Para efeito de sorteio na casa, foram consideradas somente pessoas da família, acima de 20 anos, até a lucidez; c) Quando não havia informante qualificado era programada um novo retorno quantas vezes fosse necessário; d) Somente se efetuava uma substituição nos casos em que não havia sido possível determinar o perfil da pessoa a ser entrevistada. e) $\mathrm{Na}$ ausência do sorteado na residência, era iniciado o processo de voltas racionais, até pelo menos duas voltas; f) Foram adotados 5 critérios para substituição do entrevistado como: por recusa total, cumpridas as voltas racionais, por incapacidade física ou mental, por impedimento de algum membro da família, quando o sorteado estivesse viajando ou hospitalizado inviabilizando a aplicação. As substituições eram realizadas por pessoas do mesmo sexo, faixa etária e classe social do sorteado e podiam ser obtidas nos intervalos dos domicílios sorteados; g) Nos casos de apartamentos era considerado um domicílio para efeito de contagem de pulos, que começava sempre pelo último andar, do maior número e prosseguia em ordem decrescente.

A aplicação do questionário se deu no período de abril de 1995 a julho de 1995. A equipe que desenvolveu o trabalho de campo foi composta por dois verificadores e vinte entrevistadores. Aos verificadores cabia observar se a entrevista foi realizada de maneira apropriada através da confirmação de algumas questões do questionário e ainda 
a verificação do sorteio correto dos domicílios, ou seja, se haviam sido respeitados os devidos intervalos.

Para a análise estatística foi utilizado o teste de qui-quadrado $^{23}$, com nível de significância de $\mathrm{p}<0,05$.

\section{RESULTADOS}

As tabelas de 1 a 5 mostram a distribuição dos entrevistados que estão, ou não, engajados em atividade física regular de acordo com a idade, sexo, estado civil e nível social, respectivamente.

A tabela 3 demonstra que existe uma diferença estatística significante entre homens e mulheres que praticam atividade física regular $(\mathrm{p}<0,0001)$.

Uma diferença estatística significante entre o número de indivíduos que praticam atividade física e aqueles que não a praticam foi observada $(\mathrm{p}<0,0001-$ tabela 1$)$. Do mesmo modo, uma diferença significante também foi obtida quando as queixas de distúrbios do sono comparadas entre

\section{TABELA 1}

Número total de entrevistados que estão regularmente engajados em algum tipo de atividade física, distribuídos por grupos de idade

\begin{tabular}{lc}
\hline \multicolumn{1}{c}{ Grupos } & $\begin{array}{c}\text { No de entrevistados que praticam } \\
\text { atividade física regularmente }\end{array}$ \\
I (20-30 anos) & 95 \\
II (31-40 anos) & 69 \\
III (41-50 anos) & 59 \\
IV (mais de 51 anos) & 90 \\
Total & 313 \\
\hline
\end{tabular}

TABELA 2

Número de entrevistados que praticam atividade física, divididos por idade

\begin{tabular}{lccccc}
\hline \multirow{2}{*}{ Grupos } & \multicolumn{3}{c}{ Pratica exercícios regularmente } \\
\cline { 2 - 3 } \cline { 5 - 6 } & \multicolumn{2}{c}{ Sim } & & \multicolumn{2}{c}{ Não } \\
\cline { 2 - 3 } \cline { 5 - 6 } & No & $\%$ & & No & $\%$ \\
I (20-30 anos) & 95 & 38 & & 155 & 62 \\
II (31-40 anos) & 69 & 27,8 & & 179 & 72,2 \\
III (41-50 anos) & 59 & 31,2 & & 130 & 68,8 \\
IV (mais de 51 anos) & 90 & 28,8 & & 223 & 71,2 \\
Total & 313 & 31,3 & & $687 *$ & $68,7 *$ \\
\hline
\end{tabular}

* Diferença estatisticamente significante entre o número total de indivíduos engajados em atividade física regular e o número de indivíduos que não estão engajados ( $p<$ $\left.0,0001-\chi^{2}\right)$. os indivíduos fisicamente ativos e aqueles que não praticam atividade física regular ( $\mathrm{p}<0,03$ - tabela 6).

A periodicidade da atividade física durante a semana, tipo de atividade e exercício supervisionado estão apre-

TABELA 3

Distribuição por sexo dos indivíduos entrevistados sobre a prática ou não de algum tipo de atividade física

\begin{tabular}{|c|c|c|c|c|c|c|}
\hline \multirow[t]{2}{*}{ Sexo } & \multicolumn{2}{|c|}{ Total } & \multicolumn{4}{|c|}{ Exercita regularmente } \\
\hline & № & $\%$ & $\underset{\text { Sim }}{\operatorname{Sim}}$ & $\underset{\%}{\operatorname{Sim}}$ & $\begin{array}{c}\text { Não } \\
\text { № }\end{array}$ & $\begin{array}{c}\text { Não } \\
\%\end{array}$ \\
\hline Homem & 426 & 42,6 & 174 & 55,6 & 252 & 36,7 \\
\hline Mulher & 574 & 57,4 & 139* & 44,4 & $435 *$ & 63,3 \\
\hline Total & 1.000 & 100 & 313 & 100 & 687 & 100 \\
\hline
\end{tabular}

* Diferença significante entre homens e mulheres que se exercitam regularmente $\left(* \mathrm{p}<0,0001-\chi^{2}\right)$.

TABELA 4

Distribuição de acordo com o estado civil de $\mathbf{1 . 0 0 0}$ entrevistados sobre a prática ou não de atividade física

\begin{tabular}{lcccccccc} 
Estado civil & \multicolumn{2}{c}{$\begin{array}{c}\text { Total } \\
\text { entrevistados }\end{array}$} & & \multicolumn{3}{c}{$\begin{array}{c}\text { Exercitam regularmente } \\
\text { total entrevistados }\end{array}$} \\
\cline { 2 - 3 } \cline { 6 - 8 } & No & $\%$ & & $\begin{array}{c}\text { Sim } \\
\text { No }\end{array}$ & $\begin{array}{c}\text { Sim } \\
\%\end{array}$ & $\begin{array}{c}\text { Não } \\
\text { No }\end{array}$ & $\begin{array}{c}\text { Não } \\
\%\end{array}$ \\
Casado & 590 & 59,0 & & 178 & 56,9 & 412 & 60,0 \\
Solteiro & 229 & 22,9 & & 89 & 28,4 & 140 & 20,4 \\
Viúvo & 117 & 11,7 & & 26 & 8,3 & 91 & 13,2 \\
Divorciado & 64 & 6,4 & & 20 & 6,4 & 44 & 6,4 \\
Total & 1.000 & 100 & & 313 & 100 & 687 & 100 \\
\hline
\end{tabular}

\section{TABELA 5}

Distribuição dos indivíduos que praticam atividade física regular de acordo com a classe social

\begin{tabular}{|c|c|c|c|c|c|c|}
\hline \multirow[t]{2}{*}{ Classe social } & \multicolumn{2}{|c|}{ Total } & \multicolumn{4}{|c|}{ Exercitam regularmente } \\
\hline & № & $\%$ & $\underset{\text { Noo }}{\text { Sim }}$ & $\underset{\%}{\operatorname{Sim}}$ & $\begin{array}{c}\text { Não } \\
\text { № }\end{array}$ & $\begin{array}{c}\text { Não } \\
\%\end{array}$ \\
\hline Classe A & 50 & 5,0 & 28 & 8,9 & 22 & 3,2 \\
\hline Classe B & 269 & 26,9 & 102 & 32,6 & 167 & 24,3 \\
\hline Classe C & 313 & 31,3 & 113 & 36,1 & 203 & 29,5 \\
\hline Classe D & 256 & 25,6 & 60 & 19,2 & 196 & 28,5 \\
\hline Classe E & 109 & 10,9 & 10 & 3,2 & 99 & 14,4 \\
\hline Total & 1.000 & 100 & 313 & 100 & 687 & 100 \\
\hline
\end{tabular}

Classe social determinada do maior (Classe A) para o menor ganho familiar, nível de educação e poder de compra (Classe E). 
sentadas nas tabelas 7, 8 e 9, respectivamente. Nenhuma diferença significante foi obtida nos itens acima mencionados.

\begin{tabular}{cccc}
\hline \multicolumn{3}{c}{ TABELA 6} \\
$\begin{array}{c}\text { Percentagem dos } \mathbf{1 . 0 0 0} \text { indivíduos entrevistados } \\
\text { sobre a prática de atividade física regular } \\
\text { com reclamações de problemas de sono }\end{array}$ \\
\hline $\begin{array}{c}\text { Exercitam } \\
\text { regularmente }\end{array}$ & Insônia & $\begin{array}{c}\text { Sonolência } \\
\text { excessiva }\end{array}$ & $\begin{array}{c}\text { Outros } \\
\text { problemas }\end{array}$ \\
Sim & $27,1 \%$ & $28,9 \%$ & $30,1 \%$ \\
Não & $72,9 \% *$ & $71,1 \% *$ & $69,9 \%$ \\
Total & $100 \%$ & $100 \%$ & $100 \%$ \\
\hline
\end{tabular}

* Diferença do grupo que pratica atividade física regular para o grupo que não pratica; $p<0,03\left(\chi^{2}\right)$.

TABELA 7

Freqüência semanal de atividade física reportada pelos entrevistados

\begin{tabular}{lcc}
\hline \multirow{2}{*}{ Freqüência } & \multicolumn{2}{c}{ Total } \\
\cline { 2 - 3 } & Número & Percentagem \\
Diariamente & 97 & 31,0 \\
3 a 6 vezes & 91 & 29,1 \\
1 a 2 vezes & 112 & 35,8 \\
Menos de uma vez & 13 & 4,2 \\
Total & 313 & 100 \\
\hline
\end{tabular}

TABELA 8

Tipo de atividade/exercício realizado pelos entrevistados

Tipo de atividade/ exercício

Caminhada
Ginástica
Futebol (campo ou indoor)
Natação
Levantamento de peso
Ciclismo
Corrida
Bicicleta ergométrica
Aeróbica
Exercícios na água
Dança
Basquetebol/Noleibol
Tênis/Squash
Fisioterapia
loga/Relaxamento
Artes marciais
Outras atividades
Total

Total

\begin{tabular}{rr}
\hline Número & Percentagem \\
114 & 36,6 \\
50 & 16,0 \\
54 & 17,3 \\
26 & 8,3 \\
24 & 7,7 \\
22 & 7,0 \\
18 & 5,8 \\
12 & 3,8 \\
12 & 3,8 \\
9 & 2,9 \\
8 & 2,6 \\
6 & 1,9 \\
6 & 1,9 \\
5 & 1,6 \\
4 & 1,3 \\
2 & 0,6 \\
10 & 3,2 \\
313 & 100
\end{tabular}

TABELA 9

Número de indivíduos que praticam atividade física sob supervisão de um profissional da área

\begin{tabular}{|c|c|c|c|c|c|c|}
\hline \multirow{2}{*}{$\begin{array}{c}\text { Com } \\
\text { supervisão }\end{array}$} & \multicolumn{2}{|c|}{ Total } & \multicolumn{4}{|c|}{ Exercitam regularmente } \\
\hline & № & $\%$ & $\begin{array}{c}\text { Sim } \\
\text { № }\end{array}$ & $\underset{\%}{\operatorname{Sim}}$ & $\begin{array}{c}\text { Não } \\
\text { № }\end{array}$ & $\begin{array}{c}\text { Não } \\
\%\end{array}$ \\
\hline Sim & 114 & 36,4 & 114 & 36,4 & - & - \\
\hline Não & 199 & 63,9 & - & - & 199 & 63,9 \\
\hline Total & 313 & 100 & 114 & 36,4 & 199 & 63,9 \\
\hline
\end{tabular}

\section{DISCUSSÃO}

O estudo epidemiológico realizado na cidade de São Paulo revelou que 1/3 da população realiza algum tipo de atividade física. Estes resultados são similares aos da literatura, mostrando a alta prevalência de indivíduos sedentários. Além disso, o estudo acima mencionado revela uma maior prevalência de homens fisicamente ativos quando comparados com as mulheres. E está de acordo com outros estudos, demonstrando que as categorias sociais mais altas são mais fisicamente ativas que as classes sociais mais bai$\operatorname{xas}^{6,7}$.

Nossos resultados mostram uma maior prevalência de pessoas casadas envolvidas em atividades físicas regulares. Entretanto, a baixa percentagem das pessoas que se exercitam regularmente e que contam com a supervisão de um profissional capacitado é uma fonte de preocupação. Esta supervisão deveria ser realizada por profissionais da área de Educação Física e/ou médicos, especialistas em Medicina Desportiva, no intuito de prevenir problemas que possam afetar a saúde da pessoa que se exercita.

Finalmente, nossos achados indicam que indivíduos engajados em atividades físicas regulares apresenta menor incidência de queixas relativas a insônia e a sonolência excessiva que os sedentários. Nenhuma outra queixa de distúrbio de sono foi reportada pelos entrevistados. A melhor explicação para esse achado é a falta de conhecimento da maior parte da população sobre os sintomas e os critérios de diagnósticos das desordens do sono. Uma vez que muitas desordens do sono não são "incapacitantes" ou as conseqüências de uma reduzida qualidade do sono não são imediatas, assim, tais desordenas não são reconhecidas como problemas do sono.

Em conclusão, os dados deste estudo mostram que 1/3 da população da cidade de São Paulo está engajada em algum tipo de atividade física e que apenas $36,4 \%$ possuem supervisão de um profissional qualificado. Isto leva à hipótese de que o aumento incidência da obesidade ${ }^{24}$, tendo como uma das consequiências o surgimento de indivíduos 
com apnéia obstrutiva durante o sono (SAS), e das queixas de insônia não são causadas somente pela inatividade, mas também pela falta de uma supervisão profissional durante a realização da atividade física, o que pode levar a um erro na prescrição e prática do exercício físico. Outro dado importante é a recomendação para a prática de exercícios físicos em horários que antecedem o horário de dormir, pois somente para os indivíduos que já estão adaptados com a aumento da intensidade e do volume na carga de treinamento pode-se se aceitar tal prescrição, desde que já estejam habituados a tal prática e não relatem qualquer problema com a qualidade do sono. Tal recomendação não se aplica a indivíduos iniciantes e/ou sedentários que podem apresentar um quadro de insônia de início de noite.

Portanto, o presente estudo indica a necessidade de uma mudança de hábitos na população da cidade de São Paulo no sentido de diminuir as principais queixas relacionadas à saúde do indivíduo, lembrando-se que a prática de exercícios físicos traz grandes benefícios à saúde das pessoas, mas desde que seja bem orientada e por um profissional qualificado.

\section{AGRADECIMENTOS}

Associação Fundo de Incentivo à Psicofarmacologia (AFIP).

\section{REFERÊNCIAS}

1. Ainsworth BE, Haskell WL, Leon AS, Jacobs DRJ, Montoye HJ, Sallis JF, Paffenbarger RSJ. Compendium of physical activities: classification of energy costs of human physical activities. Med Sci Sports Exerc 1993; 25:71-80.

2. Davies PSW, Gregory J, White A. Physical activity and body fatness in pre-school children. Int J Obes 1995;19:6-10.

3. Johnson ML, Burk BS, Mayer J. Relative importance of inactivity and overeating in the energy balance of obese high school girls. Am J Clin Nutr 1956;4:37-44.

4. Prentice AM, Jebb SA. Obesity in Britain: gluttony or sloth. BMJ 1995; 311:437-9.

5. Schulz LO, Schoeller DA. A compilation of total daily energy expenditures and body weights in healthy adults. Am J Clin Nutr 1994;60:67681 .

6. Dietz WH. The role of lifestyle in health: the epidemiology and consequences of inactivity. Proc Nutr Soc 1996;55:829-40.
7. Hahn RA, Teutsch SM, Rothenberg RB, Marks JS. Excess death from nine chronic diseases in the United States. JAMA 1986;264:2654-9.

8. MacAuley D. A descriptive epidemiology of physical activity from a Northen Ireland perspective. Irish J Med Sci 1994;163:228-32.

9. Siegel PZ, Brackbill RM, Heath GW. The epidemiology of walking for exercise: implications for promoting activity among sedentary groups. Am J Publ Health 1995;85:706-10.

10. Stephens T, Jacobs DR, White CC. A descriptive epidemiology of leisure time physical activity. Publ Health Rep 1985;100:147-58.

11. Stephens T, Craig CL, Ferris BF. Adult physical fitness in Canada: findings from the Canada Fitness Survey I. Can J Publ Health 1986;77:28590.

12. Bauman MD, Owen N. Habitual physical activity and cardiovascular risk factors. Med J Aust 1991;154:22-8.

13. Owen N, Bauman A. The descriptive epidemiology of sedentary lifestyle in adult Australians. Int J Epidemiol 1992;21:305-10.

14. Hasan J, Urponen H, Vuori I, Partinen M. Exercise habits and sleep in a middle-aged Finnish population. Acta Physiol Scand 1988;574:33-5.

15. Vuori I, Urponen H, Hasan J, Partinen M. Epidemiology of exercise effects on sleep. Acta Physiol Scand 1988;Suppl 574:3-7.

16. Driver HS, Meintejes AF, Rogers GG, Shapiro CM. Submaximal exercise effects on sleep patterns in young men before and after an aerobic training program. Acta Physiol Scand 1988;Suppl 574:8-13.

17. Trinder J, Montgomery I, Paxton SJ. The effect of exercise on sleep: the negative view. Acta Physiol Scand 1988;Suppl 574:14-20.

18. Youngstedt SD, O'Connor PJ, Dishman RK. The effects of acute exercise on sleep: a quantitative synthesis. Sleep 1997;20:203-14.

19. Cavalheiro JR. Investigação epidemiológica e entrevistas domiciliares. Rev Saúde Publ (São Paulo) 1981;15:543-50. [Epidemiological investigation and domiciliary interviews].

20. Del Giglio, Sandra Braz. Estudo da ocorrência de queixas de insônia, de sonolência excessiva diurna e das relativas às parassonias na população adulta da cidade de São Paulo. Doctoral Dissertation. Escola Paulista de Medicina, Department of Psychobiology, São Paulo, São Paulo, 1988. [Study of the occurrence of insomnia, daytime hypersomnolence and complains related to parasomnias in the general adult population of São Paulo City].

21. Bussad MD, Dini NP. Pesquisa de emprego e desemprego SEADE/ DIEESE: Regiões homogêneas da Grande São Paulo. Rev Fund SEADE (São Paulo) 1985;1:5-11. [Employment and unemployment survey SEADE/DIEESE: Homogeneous regions of São Paulo City].

22. ABIPEME. Proposição para um novo critério de classificação socioeconômica, mimeo, 1978. [Proposition for a new criterion of social-economic classification].

23. Siegel S. Estatística não paramétrica. São Paulo: Ed. McGraw-Hill do Brasil Ltda., 1979:350.

24. Fisberg M. Obesidade na infância e adolescência. São Paulo: Fundo Editorial Byk, 1995:157. [Obesity in childhood and adolescence]. 